\title{
AP2/ERF and R2R3-MYB family transcription factors: potential associations between temperature stress and lipid metabolism in Auxenochlorella protothecoides
}

\author{
Guanlan Xing ${ }^{1}$, Jinyu Li ${ }^{1}$, Wenli Li', Sin Man Lam², Hongli Yuan' ${ }^{1}$, Guanghou Shui ${ }^{2,3}$ and Jinshui Yang ${ }^{1 *}(\mathbb{D}$
}

\begin{abstract}
Background: Both APETALA2/Ethylene Responsive Factor (AP2/ERF) superfamily and R2R3-MYB family were from one of the largest diverse families of transcription factors (TFs) in plants, and played important roles in plant development and responses to various stresses. However, no systematic analysis of these TFs had been conducted in the green algae A. protothecoides heretofore. Temperature was a critical factor affecting growth and lipid metabolism of A. protothecoides. It also remained largely unknown whether these TFs would respond to temperature stress and be involved in controlling lipid metabolism process.
\end{abstract}

Results: Hereby, a total of six AP2 TFs, six ERF TFs and six R2R3-MYB TFs were identified and their expression profiles were also analyzed under low-temperature (LT) and high-temperature (HT) stresses. Meanwhile, differential adjustments of lipid pathways were triggered, with enhanced triacylglycerol accumulation. A co-expression network was built between these 18 TFs and 32 lipid-metabolism-related genes, suggesting intrinsic associations between TFs and the regulatory mechanism of lipid metabolism.

Conclusions: This study represented an important first step towards identifying functions and roles of AP2 superfamily and R2R3-MYB family in lipid adjustments and response to temperature stress. These findings would facilitate the biotechnological development in microalgae-based biofuel production and the better understanding of photosynthetic organisms' adaptive mechanism to temperature stress.

Keywords: Transcription factors, Acyltransferase, Cold stress, Heat stress, Lipidomics, Triacylglycerol

\section{Background}

AP2/ERF superfamily was a large family of plant transcription factors, containing one or two conserved AP2 DNA-binding domains (60-70 conserved amino acid sequences) [1]. The AP2/ERF superfamily was further classified into four families: AP2, ERF, DREB, related to ABI3 and VP1 (RAV) and Soloists [2, 3]. AP2/ERF

\footnotetext{
*Correspondence: yangjsh1999@163.com

1 State Key Laboratory of Agrobiotechnology, College of Biological Sciences, China Agricultural University, Beijing 100193, China

Full list of author information is available at the end of the article
}

transcription factors played important roles in many biological processes, including plant development, reproduction, biosynthesis of primary and secondary metabolites, as well as adaptation to biotic and abiotic stresses [1]. ERF family proteins, especially DREB (dehydration-responsive element binding) subfamily proteins, played important roles in abiotic stress. Overexpression of these proteins could improve plant stress resistance. MYB family was also a large group in plant TFs and widely distributed in plants [4]. MYB proteins were defined by a highly conserved DNAbinding domain, consisting of $1-4$ imperfect repeats

c) The Author(s) 2021. This article is licensed under a Creative Commons Attribution 4.0 International License, which permits use, sharing, adaptation, distribution and reproduction in any medium or format, as long as you give appropriate credit to the original author(s) and the source, provide a link to the Creative Commons licence, and indicate if changes were made. The images or other third party material in this article are included in the article's Creative Commons licence, unless indicated otherwise in a credit line to the material. If material is not included in the article's Creative Commons licence and your intended use is not permitted by statutory regulation or exceeds the permitted use, you will need to obtain permission directly from the copyright holder. To view a copy of this licence, visit http://creativecommons.org/licenses/by/4.0/. The Creative Commons Public Domain Dedication waiver (http://creativecommons.org/publicdomain/zero/1.0/) applies to the data made available in this article, unless otherwise stated in a credit line to the data. 
(R) of about 52 amino acids [5]. Based on the number of adjacent repeats, MYB proteins were classified into four types: 1R-MYB, R2R3-MYB, R1R2R3-MYB and 4R-MYB. R2R3-MYB TFs contained two repeats at the $\mathrm{N}$-terminal, and were the most common among these MYB family TFs [6]. R2R3-MYB TFs regulated plantspecific biological processes and participated in plant growth and development, secondary metabolism and stress response [7]. AP2/ERF superfamily and R2R3MYB family TFs had been identified and functionally characterized in many species of plants. However, it remained poorly known whether AP2/ERF and R2R3MYB TFs were associated with the response to temperature stress and transcriptional regulation of lipid metabolism in green algae.

Microalgae oil has great potential and provides an alternative to vegetable oils and fossil fuels. However, due to its high production cost, the commercial application of microalgae oil remained unsuccessful $[8,9]$. The construction of genetically engineered algal strains was expected to accelerate the commercialization of microalgae oil. However, due to the complexity of the microalgae oil synthesis pathway and the regulation mechanism, the actual regulation effects of one single gene or co-regulation effects of multi-genes on improving oil production varied among different algae. Courchesne et al. [10] proposed for the first time that genetic engineering of microalgae with high oil production could be constructed by modifying transcription factors. Hu et al. [11] found that there were 11 TFs that had potential transcriptional regulation effects on lipid metabolism in microalgae and these 11 TFs belonged to the AP2, ERF, MYB and bZIP family, respectively. Furthermore, it had been proved that NRR1 of SBP family, bZIP1 of bZIP family and ROC40 of MYB-related family could increase oil production in Chlamydomonas reinhardtii [12-14]. Li et al. [15] confirmed that NobZIP1 could increase algae's oil production without affecting its normal growth. However, the role of AP2/ERF and MYB TF family in lipid regulation of microalgae was relatively rarely reported. In fact, WEINKLED1 (WRI1) of AP2 family in Arabidopsis thaliana had been confirmed to be able to bind to the conserved AW-box sequence in the upstream region of fatty acid synthesis gene [16]. Kang et al. [17] heterologously expressed AtWRI1 from Arabidopsis thaliana in Nannochloropsis salina, which increased lipid production. PHR1 of MYB family had also been reported to have regulated lipid remodeling and triglyceride accumulation in Arabidopsis thaliana during phosphorus starvation [18]. Therefore, the systematic analysis of AP2/ERF and MYB TF families and the relationship between them and lipid metabolism would facilitate exploration of the relatively new research field of genetic engineering of algal strain.
Auxenochlorella protothecoides is a strain of unicellular microalgae that belong to the class Trebouxiophyceae (Chlorophyta) [19]. With the advances in cultivation strategies, A. protothecoides was found to be able to grow under autotrophic, heterotrophic and mixotrophic conditions, and have responded well to stress treatment, such as nitrogen starvation, addition of $\mathrm{NaCl}$ and low or high temperatures. High biomass and high lipid productivity had been achieved in A. protothecoides under heterotrophic and mixotrophic conditions not only in shake flasks, but also in large-scale bioreactors [20-26]. Temperature is a crucial factor for microalgae production [27]. Our previous studies had revealed that low and high temperatures triggered the increases of lipid contents of $A$. protothecoides at the expense of cell growth, and eventually influenced biomass and lipid productivities in different ways [28, 29]. Besides, LT and HT stresses, respectively, triggered increases of $18 \%$ and $8 \%$ in the degree of unsaturation of fatty acids. However, the degree of unsaturation of membrane glycerolipids remained unclear in $A$. protothecoides. Though the glycerolipid compositions of $A$. protothecoides UTEX 2341 had been identified [30, 31], there was a large blank in the knowledge of changes of glycerolipid profiles and glycerolipid metabolism in A. protothecoides in responses to temperature stimuli.

Transcriptomic analysis showed that the expression of AP2/ERF superfamily and R2R3-MYB family TFs changed significantly under temperature stress. Therefore, in this study, the integrative genome and transcriptome-wide analysis of AP2/ERF and R2R3-MYB TFs in A. protothecoides were conducted to obtain their molecular characterization. Glycerolipid analyses of A. protothecoides UTEX 2341 were performed under low-, normal and high-temperature conditions based on liquid chromatography-multiple reaction monitoring mass spectrometry. The analyses of expression profiles of lipid metabolismrelated genes aimed at an in-depth uncovering of the patterns of glycerolipid metabolism triggered by temperature stress. In addition, the regulatory network that controlled temperature-induced lipid adjustments was investigated. This study would facilitate the understanding of the mechanism underlying lipid adjustments of microalgae under environmental stress, the identifying of gene targets, and the construction of new algae strains with higher triacylglycerol (TAG) contents and temperature tolerance capacity by genetical engineering.

\section{Results}

Identification of A. protothecoides AP2/ERF and R2R3-MYB genes

Twelve AP2/ERF and 6 R2R3-MYB proteins were identified in A. protothecoides (Additional file 2: Table S1). There were two proteins with three-AP2 domains, four 
proteins with two AP2 domains and six proteins with a single AP2 domain. A new R2R3-MYB gene, ApMYB6, was first identified by the transcriptomic analysis and then was successfully cloned (Accession Number: MT863608). There were far fewer AP2/ERF and R2R3MYB family proteins in microalgae than in higher plants. The number of exons varied from 2 to 8 in AP2/ ERF genes (Additional file 1: Fig. S1). According to the genome annotation, there were gap regions in ApAP22, ApAP2-3 and ApAP2-5 genes, the structures of which needed to be further analyzed. For R2R3-MYB genes, the number of exons ranged from 2 to 10 , demonstrating highly different exon-intron structures even in the same subfamily.

\section{Conserved amino acid residues in AP2 and R2R3-MYB domains}

To identify the conserved amino acid residues in AP2 domain, both sequences of AP2 domains from A protothecoides and Arabidopsis AP2/ERF superfamily proteins were analyzed by multiple sequence alignments (Fig. 1a). The results showed that both $A$. protothecoides and Arabidopsis thaliana owned YRG and RAYD elements. However, there were low similarities among the sequences of three $\beta$ chains of AP2 domains. The AAEIRD motif associated with target sequence recognition was only found in $\beta-2$ chain of the AP2 domains from ApERF3 and ApERF4. However, no DREB subfamily TFs were found in A. protothecoides, and the VAEIRE motif was found in none of the ERF family members either.

Sequence alignment among MYB domains from R2R3MYB TFs of $A$. protothecoides (Fig. 1b) showed that the second and third tryptophans (W) in R2 domain were completely conserved, and the second tryptophan of R3 domain was completely conserved. However, the first tryptophan of R3 domain could be replaced by phenylalanine, and the third tryptophan could also be replaced by tyrosine. In addition to conserved tryptophan residues, other conserved amino acid residues in the MYB domains included $\mathrm{G}^{21}, \mathrm{~K}^{39}, \mathrm{C}^{41}, \mathrm{R}^{44}$ and $\mathrm{L}^{49}$ in the $\mathrm{R} 2$ domain and $E^{9}, R^{34}$ and $T^{35}$ in the $\mathrm{R} 3$ domain. Thereinto, $\mathrm{K}^{39}$ and $\mathrm{C}^{41}$ were located on the third $\alpha$-helix of the $\mathrm{R} 2$ MYB domains.

\section{Phylogenetic analyses of AP2/ERF and R2R3-MYB family proteins}

To clarify the evolutionary relationships among and classifications of $A$. protothecoides AP2/ERF superfamily proteins, 20 A. protothecoides AP2 domains and 38 representative Arabidopsis AP2 domains were phylogenetically analyzed (Fig. 2). RAV and Soloist subfamilies were not found in A. protothecoides. ApAP2-1 R1, ApAP2-2
R1, ApAP2-4 R1, ApAP2-6 R1, ApERF1 and ApERF2 were classified into the ANT R1 group. ApAP2-6 R2 was classified into the ANT R2 group. ApERF1 belonged to the AP2 R1 group. ApAP2-1 R2, ApAP2-2 R2, ApAP2-4 R2, ApAP2-5 R1-3 and ApERF6 were classified into the AP2 R2 group. ApERF3 and ApERF4 belonged to the ERF family B2 group. ApERF5 and ApAP2-3 R2-3 were classified into a new subgroup B7 in the ERF subfamily. However, ApAP2-3 R1 belonged to none of the above branches, it was classified as a new group in the AP2 family.

Phylogenetic analysis of 6 ApMYB proteins and 127 Arabidopsis R2R3-MYB proteins were carried out (Fig. 3). The results showed that ApMYB1 and AtCD5 shared high similarities and were clustered in the same cluster; ApMYB3, AtMYB88 and AtMYB124 were clustered in the same branch; ApMYB4 and ApMYB5 were close to the S25 subgroup of AtMYB proteins; ApMYB6, and S22 and S23 subgroups of AtMYB proteins were clustered together in the same branch; but ApMYB2 existed in different branches.

\section{Expression profiles of $A p A P 2, A p E R F$ and $A p M Y B$ genes responded to temperature stress}

The changes in transcript abundance of all the TF genes during LT and HT stress were quantified using RTqPCR analysis (Fig. 4 and Additional file 2: Table S2). All TF genes except for the expression of ApERF1 were not detected by qPCR, which might have been caused by its low transcriptional abundance. ApAP2-1, ApAP22, ApERF3 and ApERF5 showed high expression level during the early stage under LT stress. In addition, their expression levels were down-regulated at $168 \mathrm{~h}$, thus indicating that these four genes might have played a role in the adaptation to LT in the early stage. The expression of ApAP2-4 was induced at $168 \mathrm{~h}$, which indicated that ApAP2-4 played an important role in the adaptation to LT stress in the later stage. It was noteworthy that ApERF6 was continuously induced by LT. The expression of ApAP2-3 and ApAP2-6 was inhibited at LT, but up-regulated at $\mathrm{HT}$, which showed that these two TFs were only induced by HT stress. Under HT stress, most of these TFs showed a downward trend in expression, including ApAP2-1, ApAP2-2, ApAP2-5, ApERF1, $A p E R F 3, A p E R F 4$ and ApERF5.

Under LT stress, no obvious changes were detected in ApMYB1, ApMYB2 and ApMYB4 $\left(\left|\log _{2} \mathrm{FC}\right|<1\right)$, indicating that some members of R2R3-MYB family could not actively participate in the response to LT stress. Under HT stress, the transcription of $A p M Y B 1$ was increased at $48 \mathrm{~h}\left(\log _{2} \mathrm{FC}=0.95\right)$, indicating that ApMYB1 might have responded well to temperature stress in the early stage. The expression of ApMYB2-5 was down-regulated at 


\section{a $4-$ Arabidopsis thaliana}

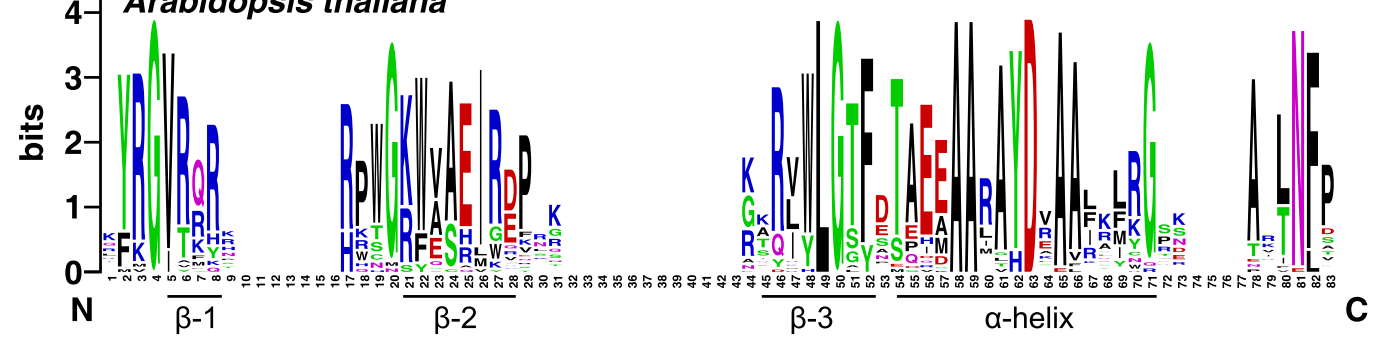

AP2

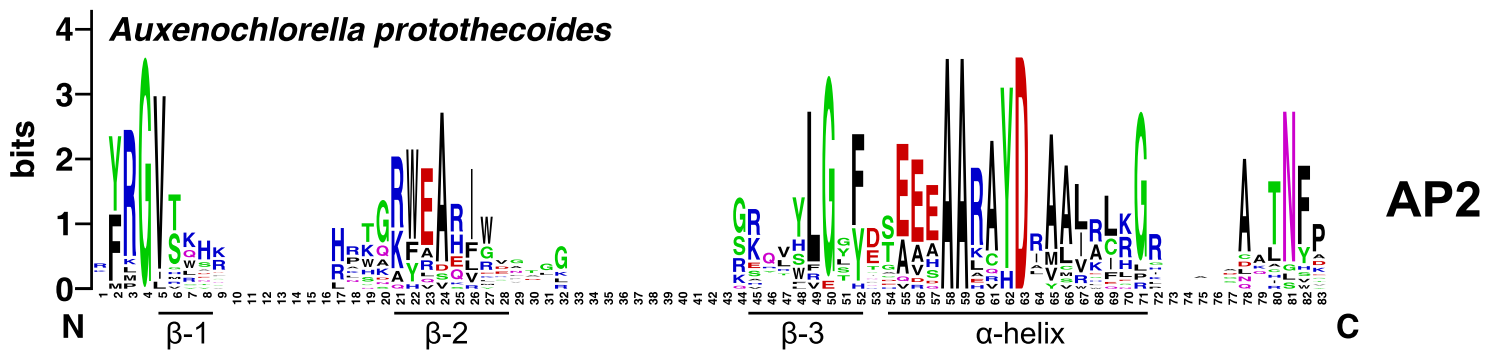

b

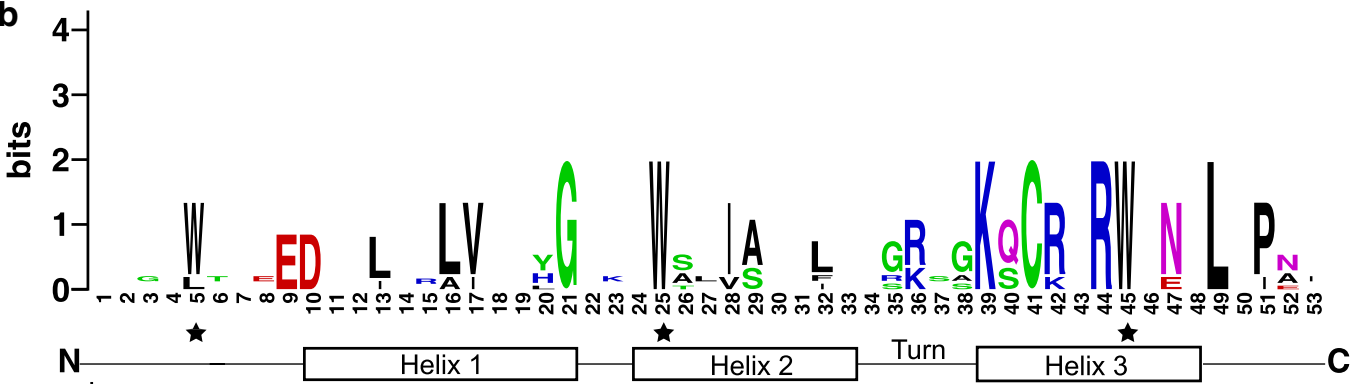

R2

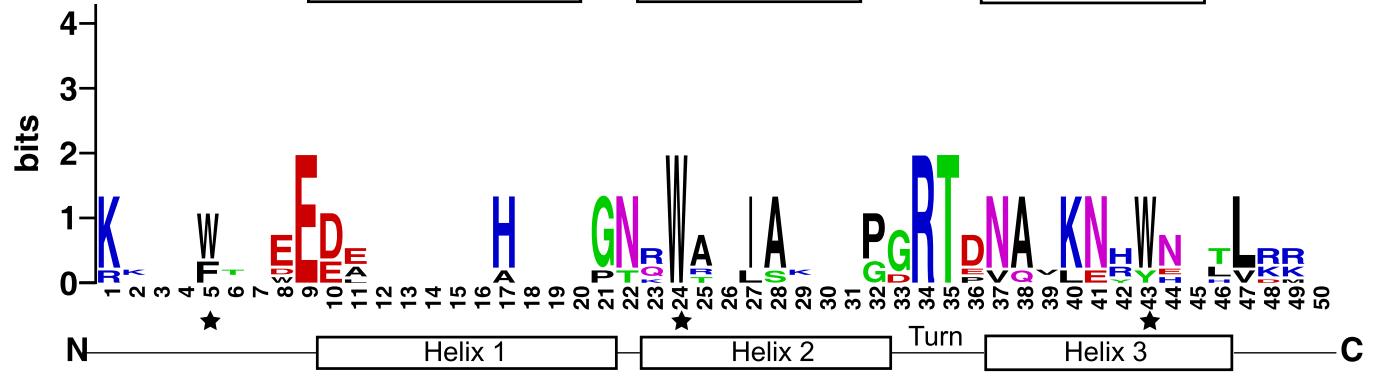

R3

Fig. 1 Sequence conservation of the conserved domain of AP2/ERF and R2R3-MYB proteins. a The sequence logos based on sequence alignments of AP2 domains of representative AP2/ERF proteins in Arabidopsis and all AP2 domains in A. protothecoides. The a-helix region and three $\beta$-sheets regions were shown. $\mathbf{b}$ The sequence logos based on sequence alignments of R2 and R3 MYB repeats of all R2R3-MYB proteins in A. protothecoides. The helix and helix-turn-helix domains that form each MYB repeat were labeled. The stars denote the typical, conserved tryptophan residues (W) in the MYB domain. The bit scores within each stack indicate the relative frequency of each amino acid at that position

$96 \mathrm{~h}$ and $168 \mathrm{~h}$ under HT stress. The similar variations of $A p M Y B 2$ and $A p M Y B 4$ genes indicated that they might have played similar roles under both LT and HT stresses.

\section{Glycerolipid profiles of $A$. protothecoides UTEX 2341}

Enhanced lipid droplet accumulation was observed in BODIPY staining observation under LT and HT stresses (Additional file 1: Fig. S2). To uncover the glycerolipid changes of A. protothecoides, the glycerolipid profiles of cells grown after 96-h incubation under three temperature conditions were generated. The lipidomic analyses showed that 287 species of glycerolipids, including 3 classes of neutral lipids, 2 classes of galactolipids, 8 classes of phospholipids, 1 class of betaine lipids, were identified in A. protothecoides UTEX 2341 (Additional file 2: Tables S3 and S4). This was the first and most 


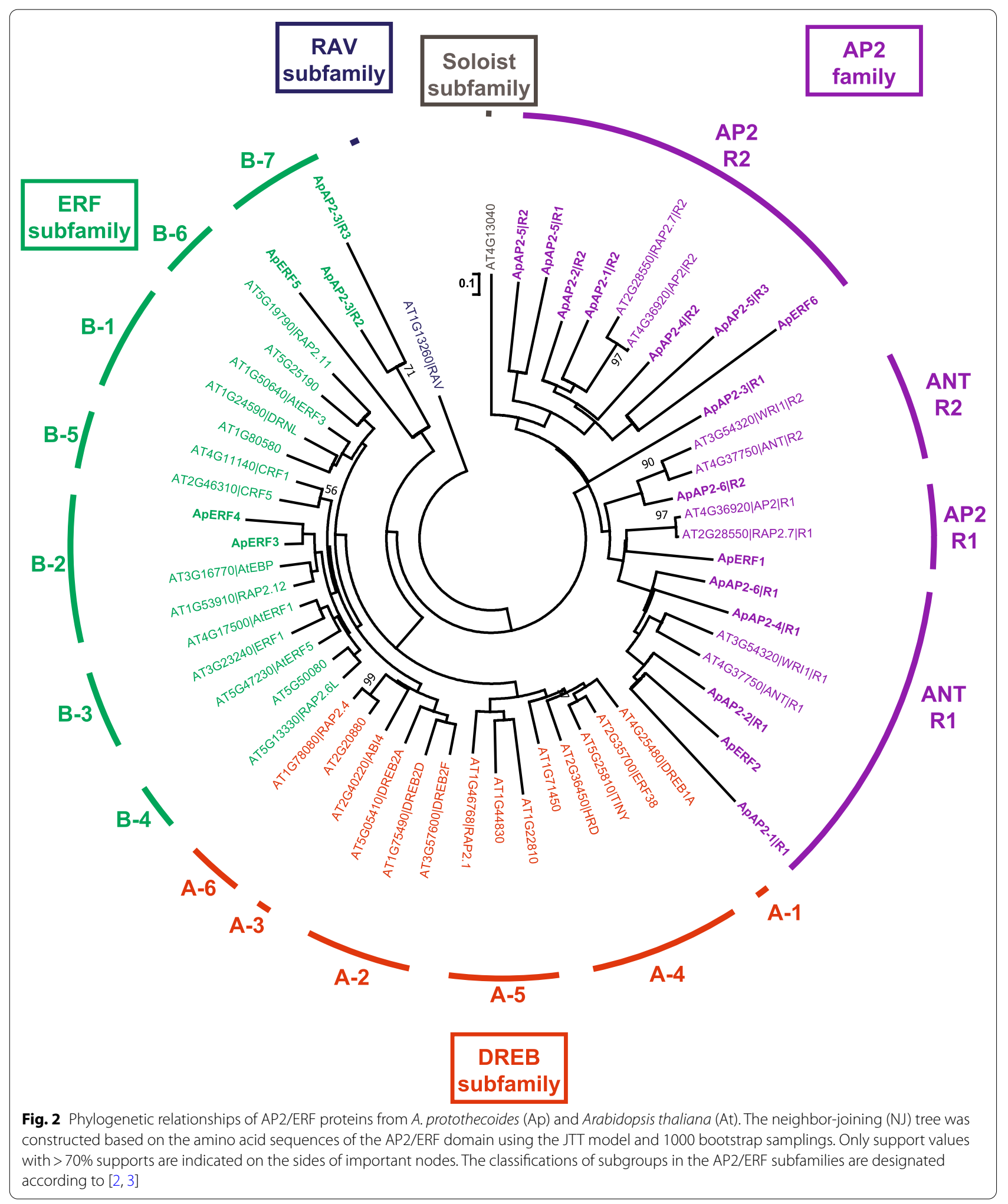




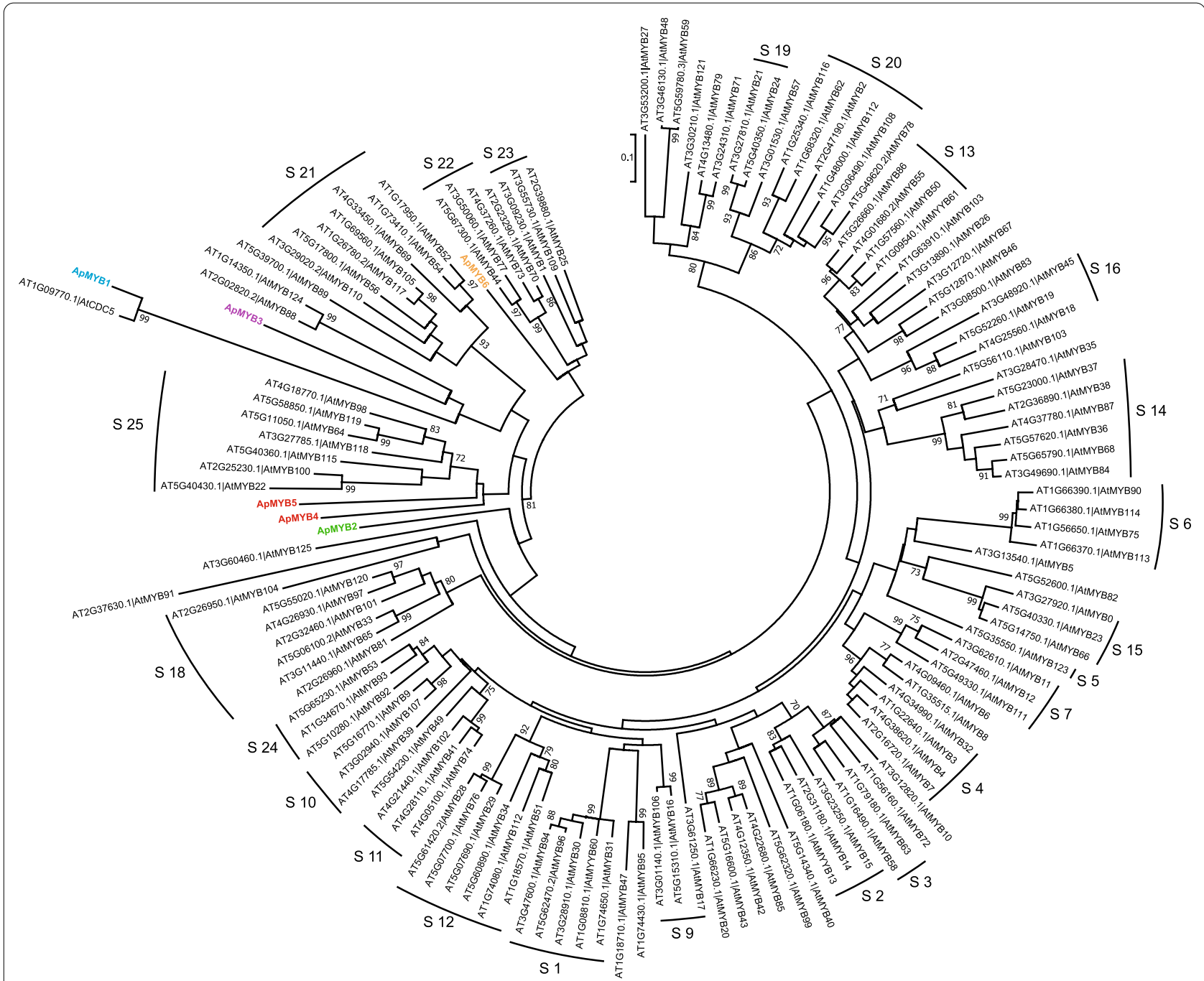

Fig. 3 Phylogenetic relationships of MYB proteins from A. protothecoides (Ap) and Arabidopsis thaliana (At). The tree was inferred based on a complete protein sequence alignment of MYB proteins using the neighbor-joining ( $\mathrm{NJ}$ ) method, JTT model and 1000 bootstraps. The numbers at the branches $>70 \%$ are shown. The classifications of subgroups are marked as previously reported [6]

detailed report of glycerolipids in A. protothecoides under three different temperature conditions. However, due to the limitation of experimental conditions, sulfolipid sulfoquinovosyldiacylglycerol (SQDG) were not analyzed. The major phospholipids included phosphatidylcholine (PC), phosphatidylinositol (PI), phosphatidylethanolamine (PE) and phosphatidylglycerol (PG), accounting for $96 \mathrm{~mol} \%$ (Additional file 1: Fig. S3). The other minor phospholipids were phosphatidylserine (PS), cardiolipin (CL), phosphatidic acid (PA) and lyso-phosphatidylethanolamine (LPE). PC accounted for more than $64 \mathrm{~mol} \%$ of phospholipids and was the most abundant phospholipid, like in most eukaryotes. The relative abundance of galactolipids was far less than that of phospholipids. The content of monogalactosyldiacylglycerol (MGDG) was the highest among the canonical photosynthetic lipids [MGDG, digalactosyldiacylglycerol (DGDG) and PG]. Though diacylglyceryltrimethylhomo-serine (DGTS) had the lowest amount of all detected glycerolipids, the sum length of two acyl chain in DGTS was the longest in all lipid species, such as those of DGTS (44:3) and DGTS (44:4).

Principal coordination analysis showed obvious distinctions among glycerolipid profiles under different temperature conditions (Additional file 1: Fig. S4). Under LT stress, the contents of TAGs were increased by 2.3 -fold and reached $68.0 \mathrm{~mol} \%$ of the total glycerolipids (Fig. 5a and Additional file 1: Fig. S3). Under HT stress, the contents of TAGs were increased by 3.4-fold and reached $75.8 \mathrm{~mol} \%$ of the total glycerolipids. These 

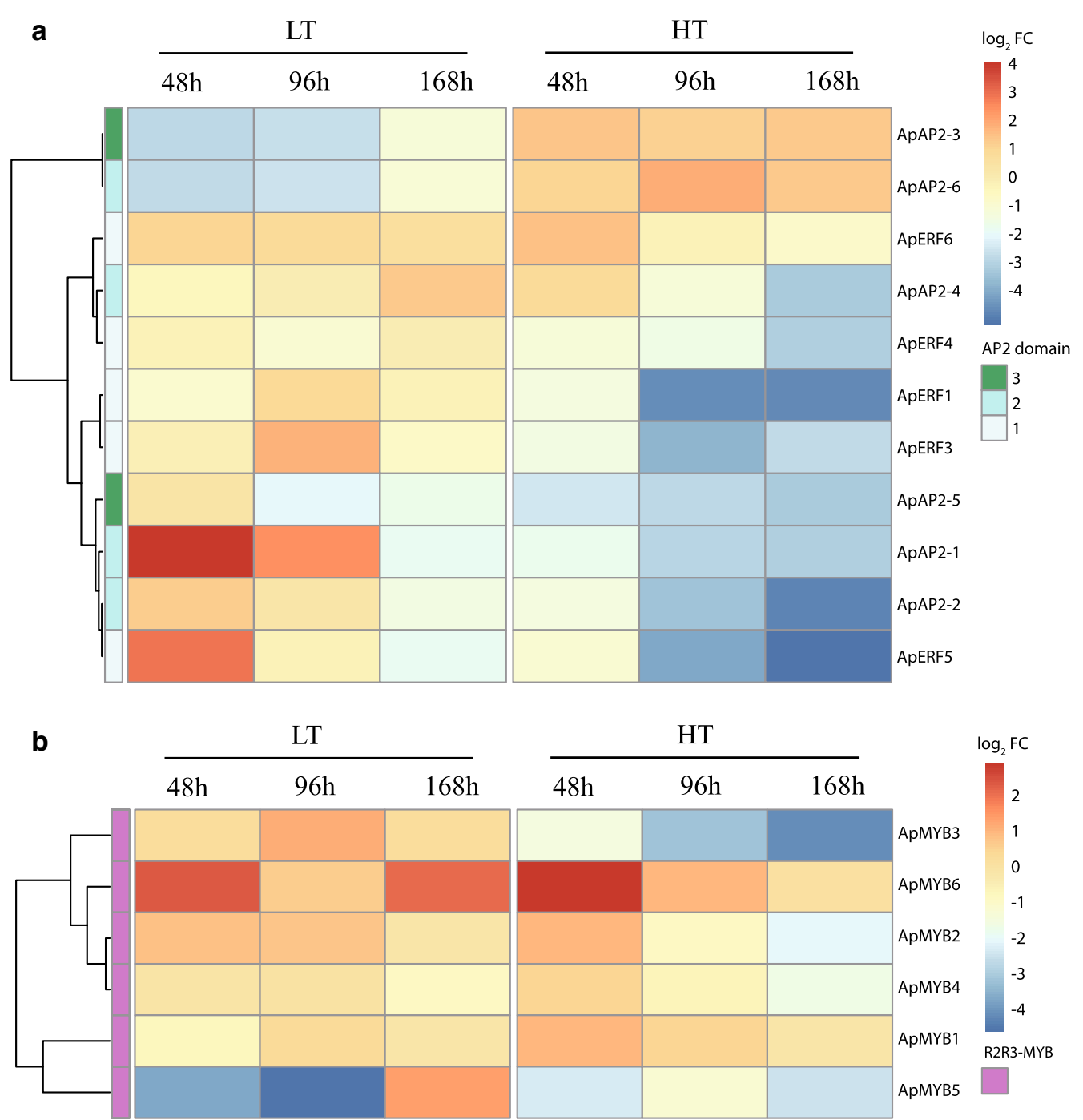

Fig. 4 Clustergram representation of transcript level of AP2/ERF (a) and R2R3-MYB (b) proteins in response to low- and high-temperature stresses in A. protothecoides grown for $168 \mathrm{~h}$. The hierarchical clustering by the Pearson rank correlation distance was conducted using the R-package 'Pheatmap'

results showed that TAGs were major products of the temperature-induced lipid metabolic reprogramming. Six polyunsaturated TAGs, including TAG52:2, TAG52:3, TAG52:4, TAG54:3, TAG54:4 and TAG54:5 accounted for between 78.2 and $83.5 \mathrm{~mol} \%$ of total TAGs (Fig. 5c). Besides, TAGs induced by LT and HT stresses showed preferences toward the biosynthesis of polyunsaturated species, such as TAG (54:5) and TAG (54:6).

The major membrane lipid classes decreased apparently in content under temperature stress, such as PC, PI, PE and MGDG (Fig. 5b and Additional file 1: Fig. S3). Under LT and HT stresses, the amounts of membrane glycerolipid species with total polar glycerolipids accounting for more than $5 \%$ decreased by different degrees. However, the amounts of the minor polar species increased, such as PS and DGTS. The contents of thylakoid membrane lipids, including DGDG and PG, decreased under HT stress. However, the content of PA significantly increased, and the proportion of DGDG in total membrane lipids increased from 3 to $5 \%$.

Under LT stress, the double-bond index (DBI) of total membrane lipids increased from 2.46 to 3.41 , and all the membrane glycerolipids classes increased apparently in DBI, from 10.5 to $97.6 \%$ (Additional file 1: Fig. S6). MGDG36:6 (18:3/18:3) accumulated to $2.53 \mathrm{nmol} \mathrm{mg}{ }^{-1}$ DW and reached $65.3 \mathrm{~mol} \%$ of MGDG (Additional file 1: Fig. S5). DGDG36:6 (18:3/18:3) accumulated to $1.32 \mathrm{nmol} \mathrm{mg} \mathrm{mg}^{-1} \mathrm{DW}$ and increased to $72.9 \mathrm{~mol} \%$ 

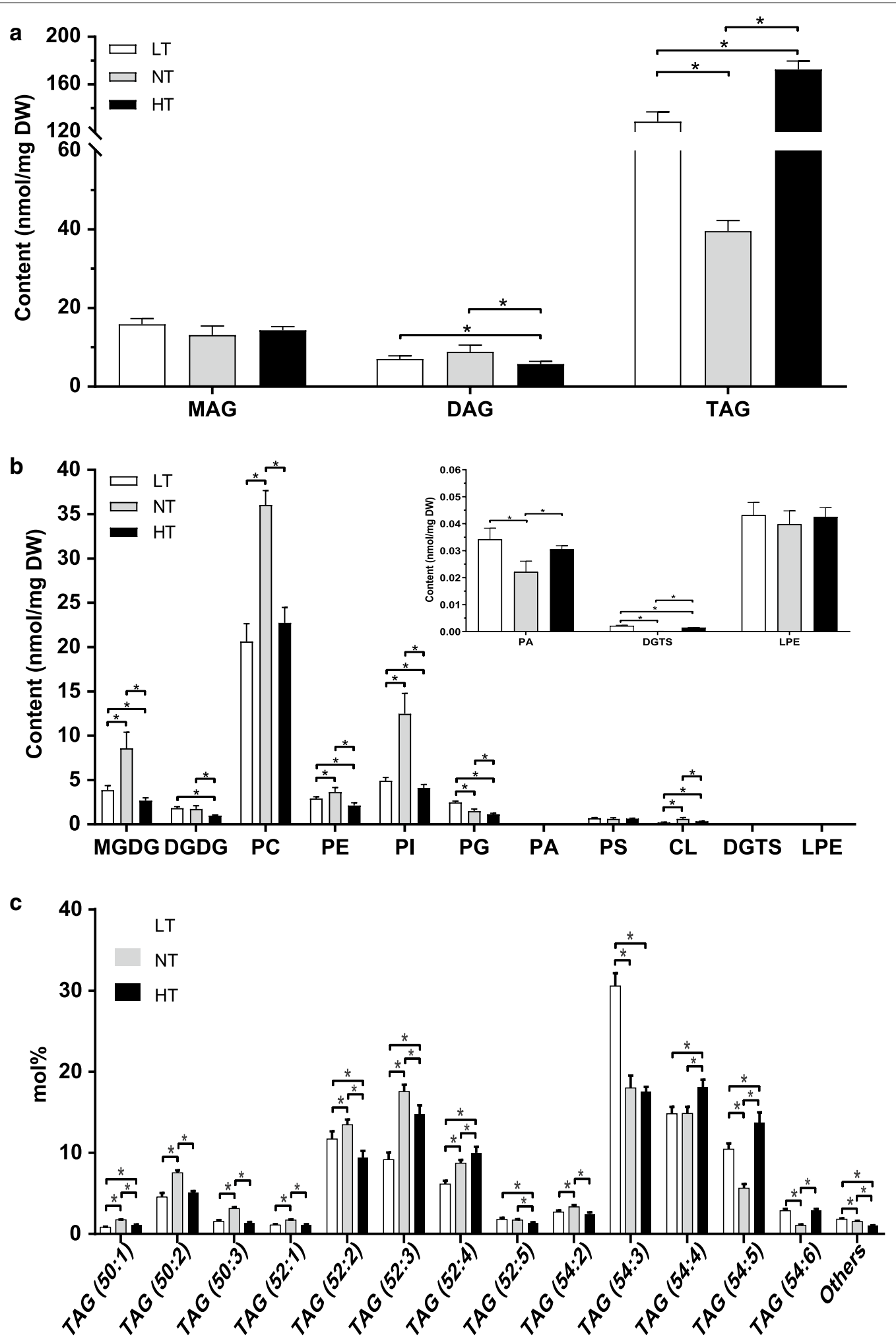

Fig. 5 Analysis of glycerolipid species in A. protothecoides grown for $96 \mathrm{~h}$ under low-, normal- and high-temperature conditions. a The content of each class of neutral lipids. $\mathbf{b}$ The content of lipids assigned with each class of galactolipids, phospholipids and betaine lipids. $\mathbf{c}$ The relative abundance of each species of TAG. All values are mean \pm SD of 4 biological repeats. $P$ values indicate significant differences between two groups (Kruskal-Wallis test: $\left.{ }^{*} P<0.05\right)$. LT low temperature, NT normal temperature, HT high temperature. The definitions of the abbreviations of lipids are shown in Additional file 2: Table S3 
of DGDG. Remarkable increases of 18:3 fatty acids in MGDG and DGDG were also observed under LT stress (Additional file 1: Fig. S7). Higher amounts of membrane lipids with $\mathrm{C} 18: 3$, which contributed to fatty acid unsaturation degrees of cell membranes, well explained why $A$. protothecoides could adapt to cold stress.

Under HT stress, the DBI of the total lipids remained relatively stable (Additional file 1: Fig. S6). The DBI of PC, one of the major membrane lipid classes, only slightly increased by $2.7 \%$. Interestingly, the DBI of galactolipids (MGDG and DGDG) significantly increased. Further analysis of the composition of galactolipids showed that the contents of $\mathrm{C} 18: 3$ in galactolipids increased significantly (Additional file 1: Fig. S7). The above results showed that HT influenced the unsaturation of membrane lipids untypically, and the responses of thylakoid membrane lipids to HT were different from those of extraplastidial lipids. This untypical change might be one of the reasons why $A$. protothecoides are unable to adapt to higher temperatures above $32{ }^{\circ} \mathrm{C}$.

\section{RNA-seq analysis of glycerolipid metabolism}

qPCR validation of the RNA-seq data was conducted in our previous study [28]. To further analyze glycerolipid metabolic adjustments at the transcript level, the pathways of glycerolipid metabolism in A. protothecoides UTEX 2341 were first constructed, and the differentially expressed genes affected by temperature stress extracted from our previous RNA-seq datasets were examined [28] (Additional file 2: Table S5). Genes with $q$-value $<0.05$ were assigned as differentially expressed genes.

Under LT stress, genes that involved in de novo TAG biosynthesis were up-regulated at the transcript level, including three glycerol-3-phosphate dehydrogenase genes (G3PDH; F751_1461, F751_3648 and F751_6745), one glycerol-3-phosphate acyltransferase gene (ATS1; F751_0154), four lysophospholipid acyltransferase genes (F751_2851, F751_2852, F751_3831 and F751_3591), one phosphatidate phosphatase gene (PPC1B; F751_1569) and one diacylglycerol acyltransferase gene (DGAT1a; F751_1386) (Fig. 6). Meanwhile, the down-regulation of the gene encoding diacylglycerol kinase (DAGK) at the transcript level was observed. Under HT stress, two G3PDH genes (F751_1461, F751_3648), two glycerol3-phosphate acyltransferase genes (ATS1 and GPAT3; F751_0154 and F751_5853), two lysophospholipid acyltransferase genes (SLC1 and LPCT4; F751_3831 and F751_2508), and PPC1B were up-regulated at the transcript level. It is noteworthy that under HT stress, only two genes encoding acyltransferase-like proteins (LCAT/ $P D A T$ and PES1) were up-regulated by 1.9 -fold and 8.2fold at the transcript level. The induction of these two genes well explained higher TAG content under HT stress than under LT stress. The results demonstrated that these two genes were closely related to enhanced TAG accumulation in $A$. protothecoides. Putative TAG, DAG and MAG lipases were found in A. protothecoides (Additional file 1: Fig. S8). Under LT and HT stresses, the transcripts of most genes involved in TAG degradation were down-regulated, which reflected depressed TAG catabolism.

Consistent with metabolite analysis of betaine lipids (Fig. 5b), the transcript level of the gene encoding DGTS synthesis enzyme (BTA1) was elevated under LT and HT stress (4.7-fold and 3.7-fold, respectively) (Fig. 6). The results suggested that DGTS biosynthesis could be induced in $A$. protothecoides under temperature stress. Under LT stress, some genes involved in PG biosynthesis exhibited a synchronized increase at the transcript level, including one phosphatidate cytidylyltransferase gene (CDS1; F751_4726), two CDP-DAG-glycerol-3-phosphate 3-phosphatidyltransferase genes (PGPS; F751_0410 and F751_5216) and one phosphatidyl glycerophosphatase gene (PGPP; F751_3869), which kept up with the increase of PG. Under HT stress, some genes of PG biosynthesis exhibited the patterns of decreased expression, including two phosphatidate cytidylyltransferase genes (CDS1 and CDSA; F751_4726 and F751_5891) and one PGPS (F751_0645).

\section{Transcriptional dynamics of lipid metabolism under low- and high-temperature stresses}

Integrated analysis of lipid metabolite and transcriptomic profiling unveiled the important candidate genes that were associated with lipid adjustment to LT and HT stresses. To gain insight into the dynamic transcriptional changes of lipid metabolism under LT and HT stresses, transcripts for 32 candidate genes involved in lipid adjustment were tested by RT-qPCR $(0 \mathrm{~h}, 48 \mathrm{~h}, 96 \mathrm{~h}$ and 168 h) (Fig. 7 and Additional file 2: Table S7).

Under LT stress, all six genes involved in de novo FA biosynthesis were up-regulated at $48 \mathrm{~h}$ and $96 \mathrm{~h}$ (Fig. 7). Thereinto, the gene encoding oleoyl-ACP thioesterase (FATA; F751_4313) remained highly expressed at $168 \mathrm{~h}$. The enhanced expression of these genes indicated that LT stress continuously activated de novo FA biosynthesis. In de novo TAG biosynthesis, GPAT3, DGAT1a and DGAT1b were up-regulated and $S L C 1$ was downregulated at the transcript level. At $48 \mathrm{~h}$ and $96 \mathrm{~h}$, only $D G A T 1 a$ in all of the three DGAT genes was up-regulated, which further indicated that DGAT1a might have played a role in early TAG accumulation and the early response to LT stress. The down-regulation of PES1 and $L C A T / P D A T$ at the transcript level indicated that TAG biosynthesis from the membrane lipid transformation could be inhibited. The decreased transcripts 


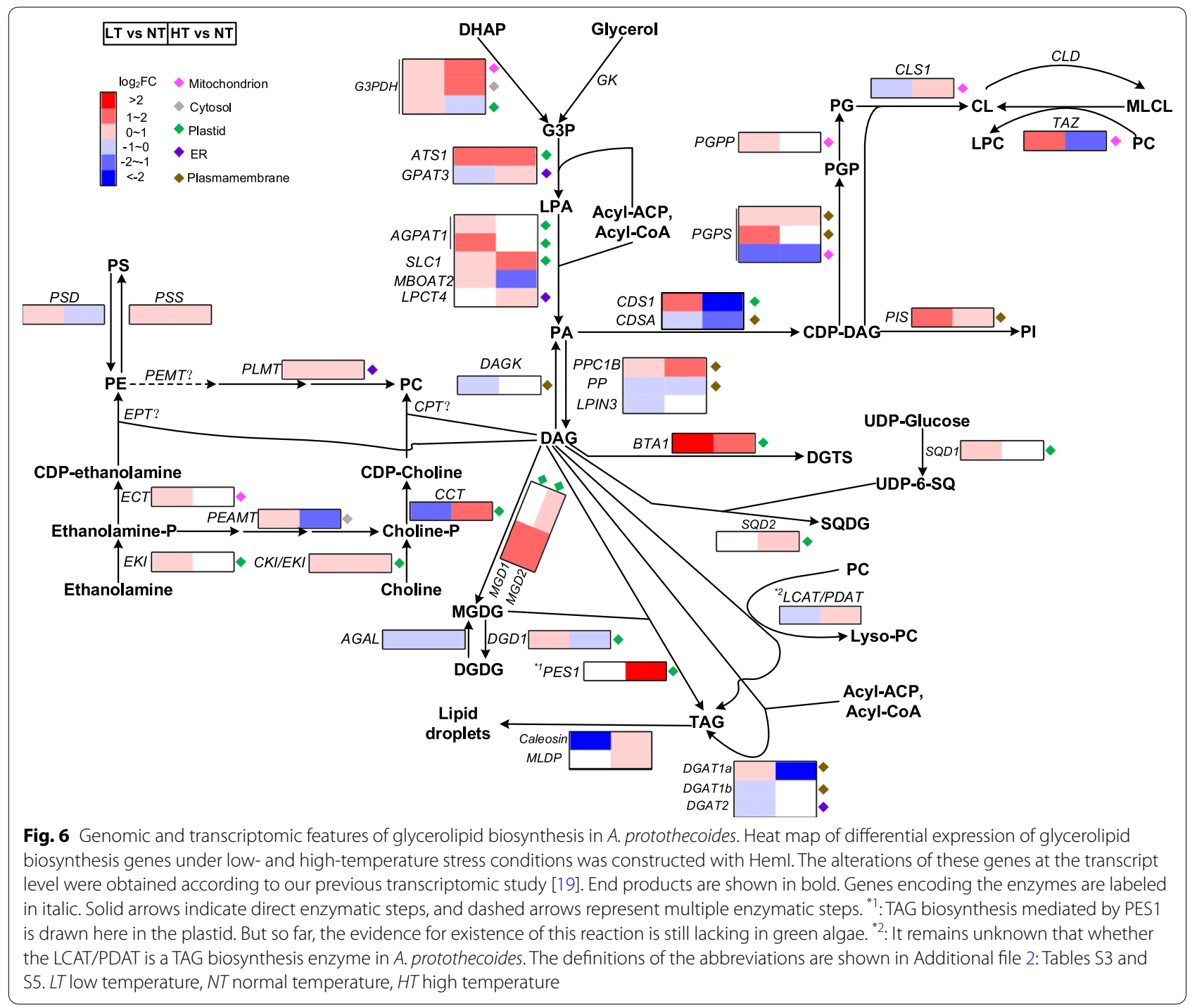

of triacylglycerol lipase sugar dependent 1 (SDP1; F751_6987) and acyl-CoA oxidase 4 (ACOX4; F751_5463) were also observed. In addition, the high expression of $\omega-3$ fatty acid desaturase (FAD7; F751_6504) was closely related to the dramatic increase of $\omega-3$ fatty acids at LT. The expression of acyl-ACP desaturase 5 (SAD1; F751_6440) was dramatically changed, suggesting that $S A D \overline{1}$ could be closely involved in the algae's adaptation to LT.

Under HT stress, the six genes involved in the de novo FA biosynthesis pathway were down-regulated (Fig. 7), but up-regulated Acetyl-CoA carboxylase (ACC ; F751_4884) and polyketide synthase (PKS; F751_4316) were observed, suggesting that HT activated cytosolic FA biosynthesis. In de novo TAG biosynthesis, up-regulated genes included LPCT4, DAGT1b and DGAT2. The increase of PES1 indicated activated TAG biosynthesis from membrane lipid transformation under HT stress. The down-regulation of CDS1, CDSA and MGDG synthase gene (MGD2; F751_2605) was also observed, suggesting the inhibited biosynthesis of phospholipids and glycolipids. Some genes involved in lipid desaturation and degradation were down-regulated, including $F A D 7$, SAD1, SDP1, ACOX4 and one $\Delta^{3,5}, \Delta^{2,4}$-dienoyl-CoA isomerase gene (DCI1; F751_6219).

\section{Establishment of lipid metabolism regulatory networks} According to the temporal changes of 17 genes encoding AP2/ERF and R2R3-MYB TFs and 32 genes involved in lipid metabolism during LT and HT stresses, the expression correlation was calculated based on the $\log _{2}$-transformed fold changes. The Pearson correlation coefficients $(|R|>0.9)$ and $P<0.05$ were used as the criteria. No positive correlation was observed in the 


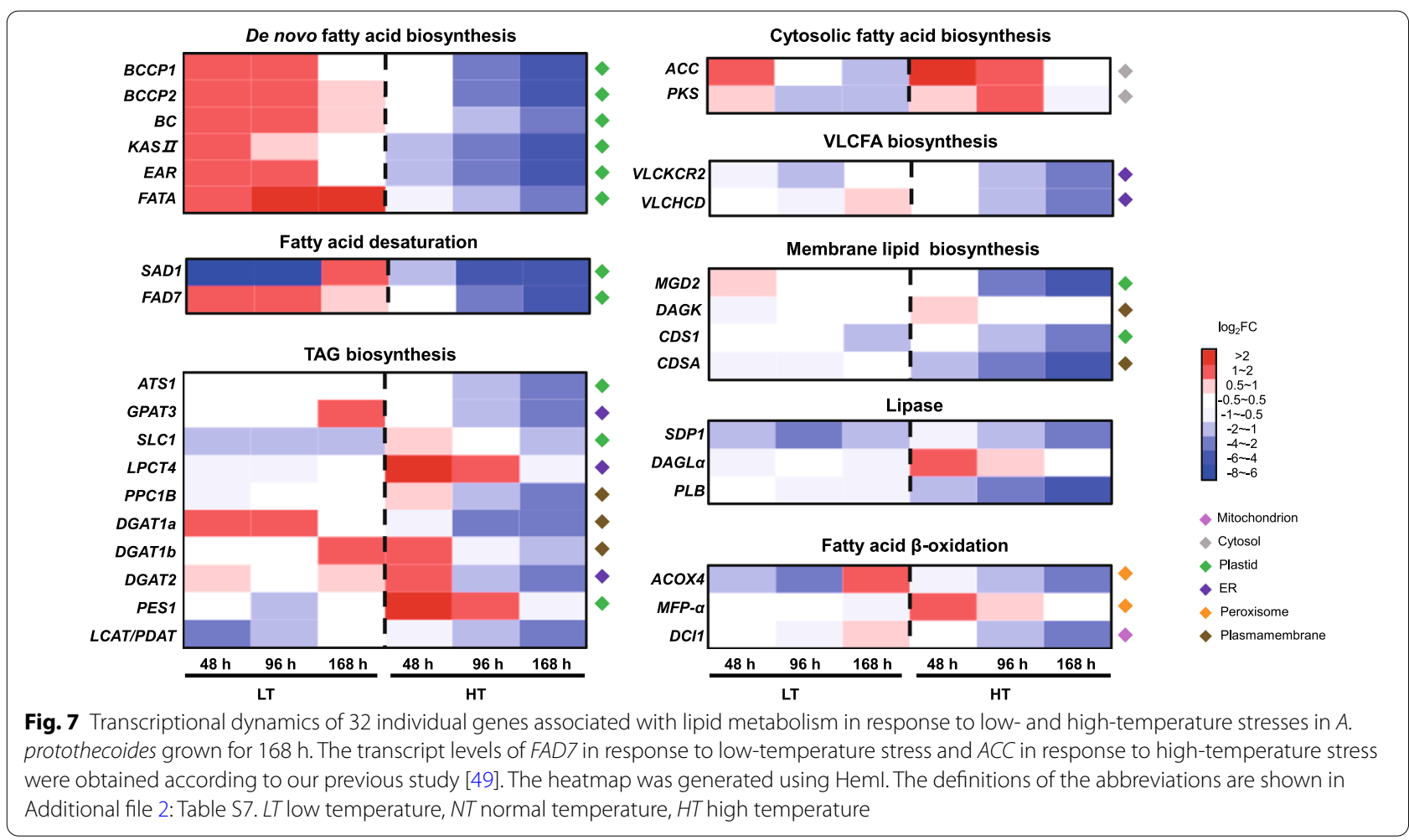

regulatory network, indicating that functions of these TFs were transcriptionally activated. The transcriptional networks consisted of 7 TFs that acted as hubs, connected with 16 putative downstream lipid-related genes (Fig. 8). ApMYB3 and ApAP2-2 had the two largest number of statistical interactions with downstream genes. Thereinto, ApMYB3 were inferred to have regulated a total of 8 genes. The results suggested that they might have served important roles in lipid adjustment and TAG accumulation. $B C P P 1$ was the most commonly regulated lipid gene by putative regulators, including $A p A P 2-2$, ApERF5, ApERF6, ApMYB2 and ApMYB3.

\section{Discussion}

Acyltransferases played a key role in the synthesis and accumulation of microalgae TAG [32]. It is well known that DGATs could catalyze the last acylation step of TAG biosynthesis, and are considered as a key enzyme that could greatly limit the inducing of TAG accumulation. In addition, heterotrophic cultivation was reported to have activated higher transcript abundance of one gene encoding DGAT and led to TAG accumulation in A. protothecoides [33]. In this study, data showed that the three DGATs in A. protothecoides varied in their responses to LT and HT stress. In Arabidopsis thaliana, DGAT1 was found to have contributed to freezing tolerance $[34,35]$. This study showed that ApDAGT1a

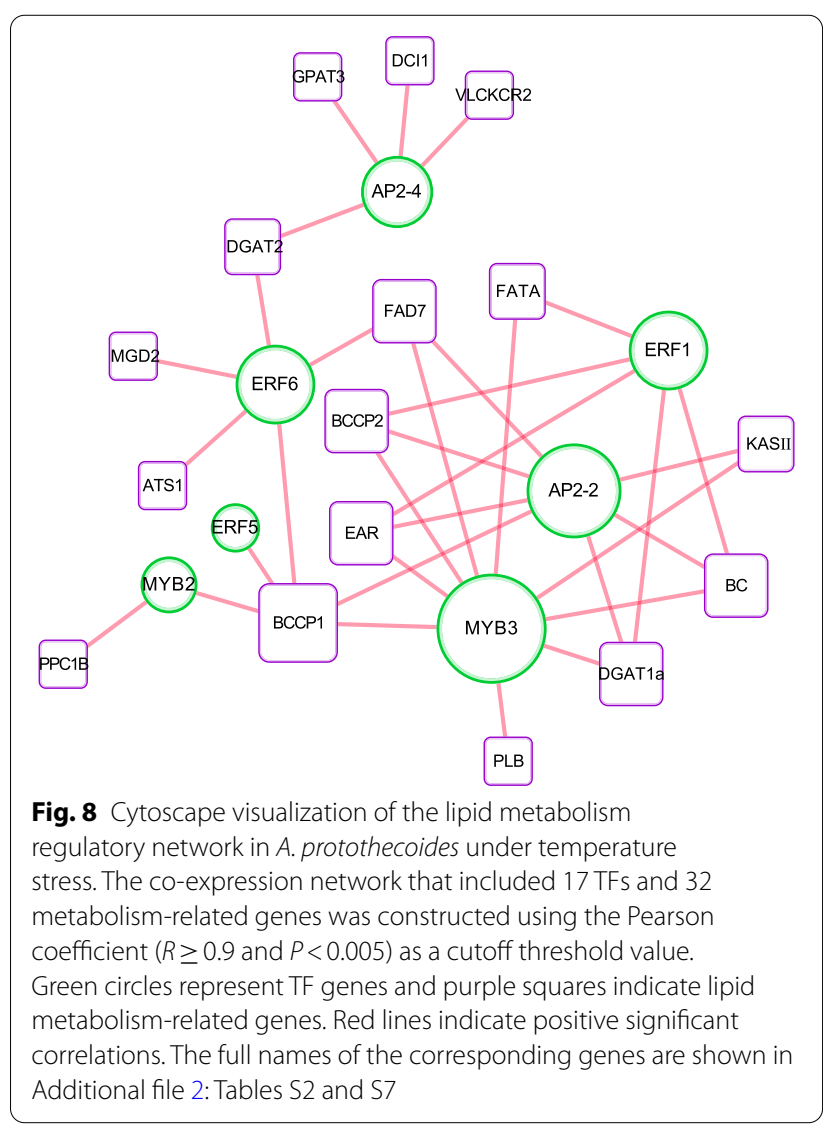


was a highly cold responsive gene, and its higher expression was closely related to TAG accumulation under LT stress. ApDAGT1b and ApDGAT2 were early heat responsive genes, and could participate in the adaption to HT stress and early TAG accumulation. The close relationship between ApDGATs and temperature stress to some extent reflected their roles in the algae's tolerance to abiotic stress, which had been neglected before. The phospholipid:diacylglycerol acyltransferase (PDAT) had been proven to have directly catalyzed the conversion of membrane lipids to TAG in Chlamydomonas reinhardtii [36]. The Chlamydomonas homologous PDAT protein was found in many microalgae [37]. Whether there were other proteins in microalgae that could directly catalyze the transformation of membrane lipids into TAG was still unclear. Except for the putative PDAT proteins, the homologs of Arabidopsis PES1 could all participate in the lipid turnover of $A$. protothecoides. The PES1 (AT1G54570) of Arabidopsis was known to possess diacylglycerol acyltransferase activities and could employ acyl-CoAs, acyl carrier proteins, and galactolipids as acyl donors to yield TAGs and had a high expression during senescence and under nitrogen deprivation [38]. By sequence alignment analysis, it was found that ApPES1 was 33.06\% consistent with PES1 from Arabidopsis thaliana, and 30.65\% consistent with PES2 from Arabidopsis thaliana in the protein sequences. Phylogenetic analysis showed that there were many PES homologous proteins in many unicellular species of green algae, including Chlorella sorokiniana, Chlamydomonas reinhardtii and Ostreococcus tauri (Additional file 1: Fig. S9). It was speculated that the theory that acyl groups from thylakoid galactolipids could directly transform to TAG mediated by PES1 might be universally applicable to green algae. It was important to note that the expression of ApPES1 was highly heat responsive. The overexpression of the putative PES1 might contribute to augments in basal thermotolerance, similar to the role of PDAT in Arabidopsis thaliana [39].

With the rapid development of next-generation sequencing technology, AP2/ERF and R2R3-MYB TFs had been widely identified in a large number of plants, and their numbers varied in different plants. In addition, some professional plant TF databases had been built to provide study tools for researchers, such as PlantTFDB and PlnTFDB. Green algae had a smaller number of AP2/ ERF and R2R3-MYB TFs, compared with Embryophyte $[40,41]$. The vast expansion of these superfamily TFs was related to more than one genome-wide duplication event during the evolution of higher plants. It was observed that $A$. protothecoides harbored only 10 AP2/ERF family TFs and no DREB subfamily TFs. The results further supported the notion that AP2/ERF superfamily TFs could originate from the AP2 family TFs and ERF subfamily TFs was the older forms of ERF family than DREB subfamily. Some TFs contained only one-AP2 domain (ApERF1 and ApERF2), which showed higher similarity to the AP2 domains contained in double/three-AP2 proteins than the other ERF TFs' AP2 domains. As mentioned in the literature review [1], a small number of one-AP2 proteins had been classified into AP2 family based on high similarities. However, plenty of studies agreed with the definition that the AP2 TFs contained only two AP2 domains. However, AP2 proteins containing more than two ( 3 and 7) AP2 domains were found in green algae [41], indicating that the traditional classification of AP2/ERF superfamily TFs needed to be revised, which would be of great help to the understanding of the evolution and functions of these TFs containing AP2 domains.

A total of five R2R3-MYB TFs were present in the $A$. protothecoides genome and one additional R2R3-MYB was found in the assembled transcriptome. Some R2R3MYB TFs of $A$. protothecoides shared high levels of sequence similarity with Arabidopsis R2R3-MYB TFs and were clustered into the known Arabidopsis functional clades. This would provide reference values to the function prediction of R2R3-MYB proteins of A. protothecoides. Prior studies had noted that the subgroup 25 of Arabidopsis R2R3-MYB family TFs played roles in seed development and nutrient distribution and storage [42-44]. For example, AtMYB96 regulated seed TAG accumulation by activating DGAT1 and PDAT1 [43]. AtMYB118 elevated the expression level of $L E A F Y$ COTYLEDON1 (LEC1), thereby promoting the expression of fatty acid biosynthetic genes $[44,45]$. Hence, it could be hypothesized that ApMYB4 and ApMYB5 were involved in the lipid biosynthetic process. The subgroup 22 of Arabidopsis R2R3-MYB family TFs, including AtMYB44, AtMYB73, and AtMYB77, were associated with stress responses, and transiently activated by cold stress [46, 47]. Based on the results of rapid up-regulation of $A p M Y B 6$ under LT and HT stress, it was speculated that $A p M Y B 6$ were involved in the early cold and heat stress responses.

Extensive studies had shown that members of AP2/ERF superfamily and R2R3-MYB family TFs were associated with the regulation of lipid metabolism in higher plants. For instance, WRINKLED1 (WRI1), one Arabidopsis AP2 family $\mathrm{TF}$, could activate the expression of fatty acid metabolic genes- $B C C P 2, K A S$ and $A C P 1$ in the early stage of seed development, and played an important role in seed maturation and oil accumulation [16, 48, 49]. Compared with AtMYB96 and AtMYB118 mentioned above, AtMYB30 could control very-long fatty acid biosynthesis [50], while AtMYB89 could repress seed lipid accumulation [51]. Studies on Jatropha curcas had shown that a 
transcription factor of R2R3-MYB family TF, JcMYB1, could bind to the promoter sequence of DGAT1 and activate the expression of targeted genes [52]. Yet the association between these two families and lipid metabolism in green algae remained largely unexplored. With the advancement of omics technologies and the application of omics-based bioinformatic analysis, AP2/ERF superfamily and R2R3-MYB family TFs that were associated with lipid biosynthesis regulation had been predicted in green algae Chlamydomonas reinhardtii [53], Chromochloris zofingiensis [54] and Nannochloropsis [11]. Besides, based on the yeast one-hybrid assay and expression correlation analysis in Chromochloris zofingiensis, $C z M Y B 1$, a R2R3 family TF was speculated to have controlled TAG biosynthesis by regulating CzDGTT5 [55]. Potential regulatory network hubs were also identified, which controlled lipid metabolism in A. protothecoides by co-expression analysis. Despite the potential association between the two families and lipid metabolism, further studies were required to confirm the functions of these TFs in green algae.

\section{Conclusion}

In this study, the genome and transcriptome-wide analyses of AP2/ERF and R2R3-MYB TFs were performed in $A$. protothecoides, and their molecular characterization and expression profiles under LT and HT stress were obtained. Lipidomic analyses showed that TAGs were major lipid products of $A$. protothecoides. Integrated analysis of lipidomic and transcriptomic profiling revealed that some lipid genes were closely related to temperature-induced lipid adjustment, especially enhanced TAG accumulation. The transcriptional dynamics analyses of important lipid metabolism-related candidate genes further revealed distinct dynamic patterns of lipid adjustment. Based on the integrated analyses of the expression profiling of the AP2/ERF and R2R3-MYB TFs and the important candidate genes, a potential regulatory network that could control the temperature-induced lipid adjustments in $A$. protothecoides was proposed.

\section{Methods}

\section{Identification of AP2/ERF and R2R3-MYB TFs in A.} protothecoides

Previously, a comprehensive transcriptomic analysis of A. protothecoides UTEX2341 under LT and HT stress was performed [28]. All unigenes were queried by iTAK program (http://itak.feilab.net/cgi-bin/itak/index.cgi) to identify and classify TFs of $A$. protothecoides [56]. Based on the combined results of the AP2 and MYB domain analyses by InterPro tool (http://www.ebi.ac.uk/inter pro/), SMART tool (http://smart.embl-heidelberg.de/), and plant transcription factor database (PlantTFDB,
http://planttfdb.gao-lab.org/index.php?sp=Apr), the candidates with less than one-AP2 domain or two MYB domains were excluded from further analysis.

\section{Sequence alignment and phylogenetic analysis}

A total of 34 representative AP2/ERF and 127 R2R3MYB protein sequences of Arabidopsis thaliana were obtained from TAIR (http://www.arabidopsis.org/). Multiple sequence alignments of AP2/ERF and MYB domains were performed with ClustalW in conjunction with MEGA 7.0 using default settings. The sequence logos for these conserved domains were produced from the multiple sequence alignment files and visualized by the WebLogo tool (http://weblogo.berkeley.edu/logo. cgi) with default parameters. Phylogenetic trees were constructed using the neighbor-joining (NJ) method by the MEGA7.0 program based on the JTT model, using a bootstrap value of 1000 with default parameters.

\section{Strains and culture}

Auxenochlorella protothecoides UTEX 2341 originated from the Culture Collection of Algae at the University of Texas (UTEX, USA). The medium composition was the same as previously described [57], including $17.5 \mathrm{~g} \mathrm{~L}^{-1}$ glucose, $13 \mathrm{~g} \mathrm{~L}^{-1}$ casein, $0.1 \mathrm{~g} \mathrm{~L}^{-1}$ yeast extract, $2 \mathrm{~g} \mathrm{~L}^{-1} \mathrm{NH}_{4} \mathrm{Cl}, 1 \mathrm{~g} \mathrm{~L}^{-1} \mathrm{KH}_{2} \mathrm{PO}_{4}, 2 \mathrm{~g} \mathrm{~L}^{-1}$ $\mathrm{Na}_{2} \mathrm{HPO}_{4}, 0.5 \mathrm{~g} \mathrm{~L}^{-1} \mathrm{MgSO}_{4} \cdot 7 \mathrm{H}_{2} \mathrm{O}, 0.01 \mathrm{~g} \mathrm{~L}^{-1} \mathrm{FeSO}_{4}$, $0.01 \mathrm{~g} \mathrm{~L}^{-1} \mathrm{CaCl}_{2}$ and $1 \mathrm{~mL} \mathrm{~L}^{-1}$ micronutrient solution [3.58 g Al $2(\mathrm{SO} 4)_{3} \cdot 18 \mathrm{H}_{2} \mathrm{O}, 12.98 \mathrm{~g} \mathrm{MnCl}_{2} \cdot 4 \mathrm{H}_{2} \mathrm{O}, 1.83 \mathrm{~g}$ $\mathrm{CuSO}_{4} \cdot 5 \mathrm{H}_{2} \mathrm{O}$ and $3.2 \mathrm{~g} \mathrm{ZnSO}_{4} \cdot 7 \mathrm{H}_{2} \mathrm{O}$ per liter of distilled water]. The inoculum cultures were incubated at $28{ }^{\circ} \mathrm{C}$ under $50 \mu \mathrm{mol} \mathrm{m}{ }^{-2} \mathrm{~s}^{-1}$ PAR in the dark/light cycles of 12-h Light/12-h Dark on an orbital shaker at $140 \mathrm{rpm}$ for $48 \mathrm{~h}$ to reach the midlog phase $\left(\times 10^{8}\right.$ cells $\left./ \mathrm{mL}\right)$ as of seeds. Then, they were inoculated at the ratio of $10 \%(\mathrm{v} / \mathrm{v})$ into a 500-mL Erlenmeyer flask with $200 \mathrm{~mL}$ medium with orbital shaking at $140 \mathrm{rpm}$ under different temperature conditions $\left(10{ }^{\circ} \mathrm{C} / 28{ }^{\circ} \mathrm{C} / 32{ }^{\circ} \mathrm{C}\right)$. Cells were cultivated without light illumination and any external supply of $\mathrm{CO}_{2}$. The experiments were repeated at least three times.

\section{Lipid extraction, LC-MS analyses, RNA extraction and RT-qPCR}

Cells were collected at $96 \mathrm{~h}$ in 10-mL tubes, rapidly harvested by centrifugation, frozen in liquid nitrogen and stored at $-80{ }^{\circ} \mathrm{C}$ until lipid extraction. The lipid inactivation and extraction steps were performed twice and lipid extracts were pooled and dried. All LC-MS analyses were carried out on an Exion UPLC coupled to QTRAP 6500 Plus (Sciex, Framingham, MA). Further details of lipid extraction and LC-MS analyses were described in the Additional file 3: Additional Methods. Total RNA extraction, cDNA synthesis and RT-qPCR were carried 
out as previously described [57]. Detailed protocols were shown in the Additional file 3: Additional Methods.

\section{Statistical analysis}

Data statistical analyses were performed using the SPSS 21 software. Data were presented as mean values \pm SDs. For the statistical analyses of lipidomic data, nonparametric comparisons were performed by applying Kruskal-Wallis test $(P<0.05)$.

\section{Supplementary Information}

The online version contains supplementary material available at https://doi. org/10.1186/s13068-021-01881-6.

Additional file 1: Figure S1. The exon-intron features of all the AP2/ ERF and R2R3-MYB genes in A. protothecoides. Yellow boxes represent the exons. Figure S2. Confocal laser microscopy of $A$. protothecoides grown for $168 \mathrm{~h}$ under low, normal and high temperature conditions. Figure S3. Analysis of glycerolipid species in A. protothecoides grown at low, normal and high temperatures after $96-\mathrm{h}$ cultivation. Figure S4. Principal coordinate analysis plot of the lipidomic data from $A$. protothecoides under three different temperature treatments. Figure S5. Changes in the content of the molecular species of membrane lipids in A. protothecoides grown for $96 \mathrm{~h}$ under low and high temperatures stress. Figure S6. The alterations of unsaturation levels of glycerolipids in A. protothecoides grown for $96 \mathrm{~h}$ under low and high temperature stress. Figure S7. Fatty acid composition changes of MGDG (A) and DGDG (B) in A. protothecoides grown for $96 \mathrm{~h}$ under low and high temperature stress. Figure S8. Heat map of differential expression of genes encoding lipases and genes involved in lipid transport under low and high temperature stress. Figure S9. Phylogenetic relationships of lipid acyltransferases from A. protothecoides, other green algae and plant.

Additional file 2: Table S1. The RNA-seq data for the putative AP2/ERF and R2R3-MYB transcriptional factors of A. protothecoides. Table S2. The relative transcript levels of $A P 2 / E R F$ and R2R3-MYB genes in response to low and high temperature stress in A. protothecoides grown for $168 \mathrm{~h}$. Table S3. The detailed information of lipid species identified in this study. Table S4. The contents of the glycerolipid molecular species in A. protothecoides grown for $96 \mathrm{~h}$ under low, normal and high temperature conditions. Table S5. The RNA-seq data for the putative genes in glycerolipid metabolism of A. protothecoides grown for $96 \mathrm{~h}$ under low and high temperature stress. Table S6. Subcellular localization predictions for candidate proteins involved in glycerolipid metabolism in A. protothecoides. Table S7. The relative transcript levels of 32 individual genes associated with lipid metabolism in response to low and high temperature stress in A. protothecoides grown for $168 \mathrm{~h}$. Table S8. Primers sequences used for RT-qPCR analysis.

Additional file 3. Additional methods.

\section{Abbreviations}

ACC: Acetyl-CoA carboxylase; ACOX: Acyl-CoA oxidase; AP2: APETALA2; BL: Betaine lipid; CDS: Phosphatidate cytidylyltransferase; CL: Cardiolipin; DAG: Diacylglycerol; DAGK: Diacylglycerol kinase; DBI: Double-bond index; DCI1: $\triangle^{3: 5}: \triangle^{2: 4}$-Dienoyl-COA isomerase; DGAT: Diacylglycerol acyltransferase; DGDG: Digalactosyldiacylglycerol; DGTS: Diacylglyceryl-N:N:N-trimethylhomoserine; DREB: Dehydration-responsive element binding; ERF: Ethylene Responsive Factor; FA: Fatty acid; FAD7: $\omega$-3 Fatty acid desaturase; FATA: Oleoyl-ACP thioesterase; G3PDH: Glycerol-3-phosphate dehydrogenase; HT: High temperature; LPE: Lyso-phosphatidylethanolamine; LT: Low temperature; MAG: Monoacylglycerol; MGD: Monogalactosyldiacylglycerol synthase; MGDG: Monogalactosyldiacylglycerol; NT: Normal temperature; PA: Phosphatidic acid; PC: Phosphatidylcholine; PDAT: Phospholipid:diacylglycerol acyltransferase; PE: Phosphatidylethanolamine; PES1: Acyltransferase-like protein; PG: Phosphatidylglycerol; PGPP: Phosphatidylglycerophosphatase; PGPS:
CDP-DAG-glycerol-3-phosphate 3-phosphatidyltransferase; Pl: Phosphatidylinositol; PKS: Polyketide synthase; PS: Phosphatidylserine; R: Repeat; RAV: DREB: related to ABI3 and VP1; SAD: Acyl-ACP desaturase 5; SDP1: Triacylglycerol lipase sugar dependent 1;TAG: Triacylglycerol; TF: Transcriptional factor.

\section{Acknowledgements}

This work was supported by the National Natural Science Foundation of China (No. 31570117).

\section{Authors' contributions}

GX: conceptualization, methodology, investigation, data curation, formal analysis, visualization and writing —original draft. JL, WL: investigation, data curation and formal analysis. SL: methodology, investigation, data curation and formal analysis. HY: conceptualization, writing - review and editing. GS: methodology, writing — review and editing. JY: conceptualization, writingreview and editing, supervision, funding acquisition and project administration. All the authors read and approved the final manuscript.

\section{Funding}

JY received funding by the National Natural Science Foundation of China (No. 31570117).

\section{Availability of data and materials}

All data used and/or analyzed during the current study were included in this article and the Additional files 1, 2.

\section{Ethics approval and consent to participate}

Not applicable.

\section{Consent for publication}

Not applicable.

\section{Competing interests}

The authors declare that they have no competing interests.

\section{Author details}

1 State Key Laboratory of Agrobiotechnology, College of Biological Sciences, China Agricultural University, Beijing 100193, China. ${ }^{2}$ State Key Laboratory of Molecular Developmental Biology, Institute of Genetics and Developmental Biology, Chinese Academy of Sciences, Beijing 100101, China. ${ }^{3}$ University of Chinese Academy of Sciences, Beijing 100049, China.

Received: 17 November 2020 Accepted: 8 January 2021

Published online: 15 January 2021

\section{References}

1. Licausi F, Ohme-Takagi M, Perata P. APETALA2/ethylene responsive factor (AP2/ERF) transcription factors: mediators of stress responses and developmental programs. New Phytol. 2013;199(3):639-49.

2. Mizoi J, Shinozaki K, Yamaguchi-Shinozaki K. AP2/ERF family transcription factors in plant abiotic stress responses. Biochim Biophys Acta. 2012;1819(2):86-96.

3. Li X, Zhang D, Gao B, Liang Y, Yang H, Wang Y, Wood AJ. Transcriptomewide identification, classification, and characterization of AP2/ERF family genes in the desert moss Syntrichia caninervis. Front Plant Sci. 2017;8:262.

4. Feller A, Machemer K, Braun EL, Grotewold E. Evolutionary and comparative analysis of MYB and bHLH plant transcription factors. Plant J. 2011;66:94-116.

5. Tombuloglu H, Kekec G, Sakcali MS, Unver T. Transcriptome-wide identification of R2R3-MYB transcription factors in barley with their boron responsive expression analysis. Mol Genet Genom. 2013;288(3-4):141-55.

6. Dubos C, Stracke R, Grotewold E, Weisshaar B, Martin C, Lepiniec L. MYB transcription factors in Arabidopsis. Trends Plant Sci. 2010;15(10):573-81.

7. Zhao K, Bartley LE. Comparative genomic analysis of the R2R3 MYB secondary cell wall regulators of Arabidopsis, poplar, rice, maize, and switchgrass. BMC Plant Biol. 2014;14:135.

8. Draaisma RB, Wijffels RH, Slegers PM, Brentner LB, Roy A, Barbosa MJ. Food commodities from microalgae. Curr Opin Biotechnol. 2013;24(2):169-77. 
9. Scott SA, Davey MP, Dennis JS, Horst I, Howe CJ, Lea-Smith DJ, Smith AG Biodiesel from algae: challenges and prospects. Curr Opin Biotechnol. 2010;21(3):277-86.

10. Courchesne NM, Parisien A, Wang B, Lan CQ. Enhancement of lipid production using biochemical, genetic and transcription factor engineering approaches. J Biotechnol. 2009;141(1-2):31-41.

11. Hu J, Wang D, Li J, Jing G, Ning K, Xu J. Genome-wide identification of transcription factors and transcription-factor binding sites in oleaginous microalgae Nannochloropsis. Sci Rep. 2014;4:5454.

12. Boyle NR, Page MD, Liu B, Blaby IK, Casero D, Kropat J, et al. Three acyltransferases and nitrogen-responsive regulator are implicated in nitrogen starvation-induced triacylglycerol accumulation in Chlamydomonas. J Biol Chem. 2012;287(19):15811-25.

13. Yamaoka Y, Shin S, Choi BY, Kim H, Jang S, Kajikawa M, et al. The bZIP1 transcription factor regulates lipid remodeling and contributes to ER stress management in Chlamydomonas reinhardtii. Plant Cell. 2019;31(5):1127-40.

14. Goncalves EC, Koh J, Zhu N, Yoo MJ, Chen S, Matsuo T, et al. Nitrogen starvation-induced accumulation of triacylglycerol in the green algae: evidence for a role for ROC 40 , a transcription factor involved in circadian rhythm. Plant J. 2016;85(6):743-57.

15. Li DW, Balamurugan S, Yang YF, Zheng JW, Huang D, Zou LG, Yang WD, Liu JS, Guan Y, Li HY. Transcriptional regulation of microalgae for concurrent lipid overproduction and secretion. Sci Adv. 2019;5(1):eaau3795.

16. Maeo K, Tokuda T, Ayame A, Mitsui N, Kawai T, Tsukagoshi H, Ishiguro S, Nakamura K. An AP2-type transcription factor, WRINKLED1, of Arabidopsis thaliana binds to the AW-box sequence conserved among proximal upstream regions of genes involved in fatty acid synthesis. Plant $\mathrm{J}$. 2009;60(3):476-87.

17. Kang NK, Kim EK, Kim YU, Lee B, Jeong WJ, Jeong BR, et al. Increased lipid production by heterologous expression of AtWRI1 transcription factor in Nannochloropsis salina. Biotechnol Biofuels. 2017;10:231.

18. Pant BD, Burgos A, Pant P, Cuadros-Inostroza A, Willmitzer L, Scheible WR. The transcription factor PHR1 regulates lipid remodeling and triacylglycerol accumulation in Arabidopsis thaliana during phosphorus starvation. J Exp Bot. 2015;66(7):1907-18.

19. Huerlimann R, Heimann K. Comprehensive guide to acetyl-carboxylases in algae. Crit Rev Biotechnol. 2013;33(1):49-65.

20. Xiao Y, Lu Y, Dai J, Wu Q. Industrial fermentation of Auxenochlorella protothecoides for production of biodiesel and its application in vehicle diesel engines. Front Bioeng Biotechnol. 2015;3:164.

21. Patel A, Matsakas L, Rova U, Christakopoulos P. Heterotrophic cultivation of Auxenochlorella protothecoides using forest biomass as a feedstock for sustainable biodiesel production. Biotechnol Biofuels. 2018;11(1):169.

22. Rismani-Yazdi H, Hampel KH, Lane CD, Kessler BA, White NM, Moats KM, Thomas Allnutt FC. High-productivity lipid production using mixed trophic state cultivation of Auxenochlorella (Chlorella) protothecoides. Bioprocess Biosyst Eng. 2015;38(4):639-50.

23. Xiong W, Li X, Xiang J, Wu Q. High-density fermentation of microalga Chlorella protothecoides in bioreactor for microbio-diesel production. Appl Microbiol Biotechnol. 2008;78(1):29-36.

24. Xu H, Miao X, Wu Q. High quality biodiesel production from a microalga Chlorella protothecoides by heterotrophic growth in fermenters. J Biotechnol. 2006;126(4):499-507.

25. Yang J, Rasa E, Tantayotai P, Scow KM, Yuan H, Hristova KR. Mathematical model of Chlorella minutissima UTEX2341 growth and lipid production under photoheterotrophic fermentation conditions. Bioresour Technol. 2011;102(3):3077-82.

26. Li Z, Yuan H, Yang J, Li B. Optimization of the biomass production of oil algae Chlorella minutissima UTEX2341. Bioresour Technol. 2011;102(19):9128-34.

27. Ras M, Steyer J-P, Bernard O. Temperature effect on microalgae: a crucial factor for outdoor production. Rev Environ Sci Bio. 2013;12(2):153-64.

28. Xing G, Yuan H, Yang J, Li J, Gao Q, Li W, Wang E. Integrated analyses of transcriptome, proteome and fatty acid profilings of the oleaginous microalga Auxenochlorella protothecoides UTEX 2341 reveal differential reprogramming of fatty acid metabolism in response to low and high temperatures. Algal Res. 2018;33:16-27.

29. Cao J, Yuan H, Li B, Yang J. Significance evaluation of the effects of environmental factors on the lipid accumulation of Chlorella minutissima
UTEX 2341 under low-nutrition heterotrophic condition. Bioresour Technol. 2014;152:177-84.

30. Tsugawa H, Cajka T, Kind T, Ma Y, Higgins B, Ikeda K, Kanazawa M, VanderGheynst J, Fiehn O, Arita M. MS-DIAL: data-independent MS/MS deconvolution for comprehensive metabolome analysis. Nat Methods. 2015;12(6):523-6

31. Higgins BT, Nobles D, Ma Y, Wikoff W, Kind T, Fiehn O, Brand J, VanderGheynst JS. Informatics for improved algal taxonomic classification and research: a case study of UTEX 2341. Algal Res. 2015:12:545-9.

32. Klok AJ, Lamers PP, Martens DE, Draaisma RB, Wijffels RH. Edible oils from microalgae: insights in TAG accumulation. Trends Biotechnol. 2014;32(10):521-8.

33. Gao C, Wang Y, Shen Y, Yan D, He X, Dai J, Wu Q. Oil accumulation mechanisms of the oleaginous microalga Chlorella protothecoides revealed through its genome, transcriptomes, and proteomes. BMC Genom. 2014;15:582.

34. Arisz SA, Heo JY, Koevoets IT, Zhao T, van Egmond P, Meyer J, Zeng W, Niu $X$, Wang B, Mitchell-Olds T, et al. Diacylglycerol acyltransferase 1 contributes to freezing tolerance. Plant Physiol. 2018;177(4):1410-24.

35. Tan WJ, Yang YC, Zhou Y, Huang LP, Xu L, Chen QF, Yu LJ, Xiao S. Diacylglycerol acyltransferase and diacylglycerol kinase modulate triacylglycerol and phosphatidic acid production in the plant response to freezing stress. Plant Physiol. 2018;177(3):1303-18.

36. Yoon K, Han D, Li Y, Sommerfeld M, Hu Q. Phospholipid:diacylglycerol acyltransferase is a multifunctional enzyme involved in membrane lipid turnover and degradation while synthesizing triacylglycerol in the unicellular green microalga Chlamydomonas reinhardtii. Plant Cell. 2012;24(9):3708-24

37. Zienkiewicz K, Du ZY, Ma W, Vollheyde K, Benning C. Stress-induced neutral lipid biosynthesis in microalgae-molecular, cellular and physiological insights. Biochim Biophys Acta. 2016;1861(9):1269-81.

38. Lippold F, vom Dorp K, Abraham M, Holzl G, Wewer V, Yilmaz JL, Lager I, Montandon C, Besagni C, Kessler F, et al. Fatty acid phytyl ester synthesis in chloroplasts of Arabidopsis. Plant Cell. 2012;24(5):2001-14.

39. Mueller SP, Unger M, Guender L, Fekete A, Mueller MJ. Phospholipid:diacylglycerol acyltransferase-mediated triacylglyerol synthesis augments basal thermotolerance. Plant Physiol. 2017;175:486-97.

40. Du H, Liang Z, Zhao S, Nan MG, Tran LS, Lu K, Huang YB, Li JN. The evolutionary history of R2R3-MYB proteins across 50 eukaryotes: new insights into subfamily classification and expansion. Sci Rep. 2015;5:11037.

41. Song X, Wang J, Ma X, Li Y, Lei T, Wang L, Ge W, Guo D, Wang Z, Li C, et al. Origination, expansion, evolutionary trajectory, and expression bias of AP2/ERF superfamily in Brassica napus. Front Plant Sci. 2016;7:1186.

42. Feng $S$, Xu Y, Yang L, Sun S, Wang D, Chen X. Genome-wide identification and characterization of R2R3-MYB transcription factors in pear. Sci Hortic. 2015:197:176-82.

43. Lee HG, Kim H, Suh MC, Kim HU, Seo PJ. The MYB96 transcription factor regulates triacylglycerol accumulation by activating DGAT1 and PDAT1 expression in Arabidopsis seeds. Plant Cell Physiol. 2018;59(7):1432-42.

44. Wang X, Niu QW, Teng C, Li C, Mu J, Chua NH, Zuo J. Overexpression of PGA37/MYB118 and MYB115 promotes vegetative-to-embryonic transition in Arabidopsis. Cell Res. 2009;19(2):224-35.

45. Mu J, Tan H, Zheng Q, Fu F, Liang Y, Zhang J, Yang X, Wang T, Chong K, Wang $X$-J, et al. LEAFY COTYLEDON1 is a key regulator of fatty acid biosynthesis in Arabidopsis. Plant Physiol. 2008;148(2):1042-54.

46. Jung C, Seo JS, Han SW, Koo YJ, Kim CH, Song SI, Nahm BH, Choi YD, Cheong JJ. Overexpression of AtMYB44 enhances stomatal closure to confer abiotic stress tolerance in transgenic Arabidopsis. Plant Physiol. 2008;146(2):623-35.

47. Fowler S, Thomashow MF. Arabidopsis transcriptome profiling indicates that multiple regulatory pathways are activated during cold acclimation in addition to the CBF cold response pathway. Plant Cell. 2002;14(8):1675-90

48. Baud S, Wuilleme S, To A, Rochat C, Lepiniec L. Role of WRINKLED1 in the transcriptional regulation of glycolytic and fatty acid biosynthetic genes in Arabidopsis. Plant J. 2009;60(6):933-47.

49. Cernac A, Benning C. WRINKLED1 encodes an AP2/EREB domain protein involved in the control of storage compound biosynthesis in Arabidopsis. Plant J. 2004;40(4):575-85.

50. Raffaele S, Vailleau F, Leger A, Joubes J, Miersch O, Huard C, Blee E, Mongrand S, Domergue F, Roby D. A MYB transcription factor regulates 
very-long-chain fatty acid biosynthesis for activation of the hypersensitive cell death response in Arabidopsis. Plant Cell. 2008;20(3):752-67.

51. Li D, Jin C, Duan S, Zhu Y, Qi S, Liu K, Gao C, Ma H, Zhang M, Liao Y, et al. MYB89 transcription factor represses seed oil accumulation. Plant Physiol. 2017;173(2):1211-25.

52. Khan K, Kumar V, Niranjan A, Shanware A, Sane VA. JCMYB1, a Jatropha R2R3 MYB transcription factor, modulates lipid biosynthesis in transgenic plants. Plant Cell Physiol. 2018;60:462-75.

53. Gargouri M, Park JJ, Holguin FO, Kim MJ, Wang H, Deshpande RR, Shachar-Hill Y, Hicks LM, Gang DR. Identification of regulatory network hubs that control lipid metabolism in Chlamydomonas reinhardtii. J Exp Bot. 2015;66(15):4551-66.

54. Liu J, Sun Z, Mao X, Gerken H, Wang X, Yang W. Multi-omics analysis reveals distinct mechanism of oleaginousness in the emerging model alga Chromochloris zofingiensis. Plant J. 2019;98:1060-77.

55. Mao X, Wu T, Kou Y, Shi Y, Zhang Y, Liu J. Characterization of type I and type II diacylglycerol acyltransferases from the emerging model alga
Chlorella zofingiensis reveals their functional complementarity and engineering potential. Biotechnol Biofuels. 2019;12:28.

56. Zheng Y, Jiao C, Sun H, Rosli HG, Pombo MA, Zhang P, Banf M, Dai X, Martin GB, Giovannoni JJ, et al. iTAK: a program for genome-wide prediction and classification of plant transcription factors, transcriptional regulators, and protein kinases. Mol Plant. 2016;9(12):1667-70.

57. Xing G, Liu K, Li W, Li J, Xing C, Yuan H, Yang J. Evaluation of internal reference genes in Auxenochlorella protothecoides under continuous heterotrophic culture conditions at normal, low and high temperatures. Algal Res. 2020;49:101941.

\section{Publisher's Note}

Springer Nature remains neutral with regard to jurisdictional claims in published maps and institutional affiliations.
Ready to submit your research? Choose BMC and benefit from:

- fast, convenient online submission

- thorough peer review by experienced researchers in your field

- rapid publication on acceptance

- support for research data, including large and complex data types

- gold Open Access which fosters wider collaboration and increased citations

- maximum visibility for your research: over $100 \mathrm{M}$ website views per year

At BMC, research is always in progress.

Learn more biomedcentral.com/submissions 